\title{
Suppression of superconductivity in granular metals
}

\author{
I. S. Beloborodov, A. V. Lopatin, and V. M. Vinokur \\ Materials Science Division, Argonne National Laboratory, Argonne, Illinois 60439
}

(Dated: November 21, 2018)

\begin{abstract}
We investigate the suppression of the superconducting transition temperature due to Coulomb repulsion in granular metallic systems at large tunneling conductance between the grains, $g_{T} \gg 1$. We find the correction to the superconducting transition temperature for $3 D$ granular samples and films. We demonstrate that depending on the parameters of superconducting grains, the corresponding granular samples can be divided into two groups: (i) the granular samples that belong to the first group may have only insulating or superconducting states at zero temperature depending on the bare intergranular tunneling conductance $g_{T}$, while (ii) the granular samples that belong to the second group in addition have an intermediate metallic phase where superconductivity is suppressed while the effects of the Coulomb blockade are not yet strong.

PACS numbers: 74.81.Bd, 74.78.Na, 73.40.Gk
\end{abstract}

A great deal of current experimental and theoretical research in mesoscopic physics focuses on properties of inhomogeneous granular superconductors [1, 2, 3, 4]. The interest is motivated not only by their unusual properties such as negative magnetoresistance [5], but, even to a higher extent, by the fact that they represent an exemplary disordered electronic system with experimentally accessible tunable parameters. A specific feature of granular metallic materials is the important role of the Coulomb interaction that strongly affects their behavior. The interplay between the Coulomb interaction effects and disorder was shown to suppress an electron transport in granular metals [6, 7, 8]; the fundamental question that remains open is how the Coulomb repulsion affects the superconducting properties of granular metals, and in particular, the superconducting transition temperature.

Coulomb interactions decrease the critical temperature, this effect is amplified significantly in the presence of disorder and becomes especially pronounced in lower dimensions. For example in $2 D$ (disordered) superconducting films the effect of Coulomb repulsions is known to noticeably decrease the critical temperature 9, 10, 11, 12]. The mechanism of suppression of the transition temperature can be understood as a result of the renormalization of the electron interaction in the Cooper channel by the Coulomb repulsion in the presence of scattering by impurities 13]. Since in granular samples both disorder and the strong Coulomb repulsion effects present, one expects that similar mechanism governs the reduction of the superconducting critical temperate and becomes even more pronounced for granular superconductors.

In this Letter we investigate the influence of Coulomb repulsion on the superconducting transition temperature of granular metals and demonstrate that depending on the parameters of the grains, the granular materials can be divided into two groups: (i) materials that may be found in either insulating or superconducting state at zero temperature and (ii) materials that can, in addition, exhibit an intermediate metallic state. The parameter that governs the corresponding behavior is the tunneling conductance $g_{T}$. The transport properties of granular metals were recently extensively studied in Ref. [6, 7, 8]. It was shown that for $3 \mathrm{D}$ samples there is a critical value of the bare tunneling conductance [8]

$$
g_{T}^{C}=(1 / 6 \pi) \ln \left(E_{C} / \delta\right),
$$

where $E_{C}$ is the Coulomb energy and $\delta$ is the mean level spacing in a single grain, that allows to classify granular samples by their transport properties: samples with $g_{T}<$ $g_{T}^{C}$ are insulators at zero temperature while samples with $g_{T}>g_{T}^{C}$ are metals. We show that in order to classify 3D superconducting granular samples with respect to their possible ground state at zero temperature, an additional characteristic conductance $g_{T}^{*}$ should be introduced:

$$
g_{T}^{*} \approx \frac{A}{\pi} \ln ^{2}\left(E_{C} / T_{c}^{0}\right)
$$

where $T_{c}^{0}$ is the superconducting critical temperature of a single grain and $A$ is the numerical coefficient defined below. We demonstrate that granular superconductors can be conveniently classified depending on the relation between $g_{T}^{C}$ and $g_{T}^{*}$ into two groups:

(i) Group A: Granular samples with $g_{T}^{*}<g_{T}^{C}$ may have only two phases at zero temperature depending on the tunneling conductance $g_{T}$. They are either superconductors if $g_{T}>g_{T}^{*}$, or insulators if $g_{T}<g_{T}^{*}$.

(ii) Group B: Samples with $g_{T}^{*}>g_{T}^{C}$ can be found in three phases. They are superconductors if $g_{T}>g_{T}^{*}$, insulators if $g_{T}<g_{T}^{C}$, or metals in the intermediate region $g_{T}^{*}>g_{T}>g_{T}^{C}$.

Thus, in the latter case there exists an intermediate metallic region $g_{T}^{*}>g_{T}>g_{T}^{C}$ where the superconductivity is suppressed by the Coulomb interaction while effects of Coulomb blockade are not yet strong.

Presenting our final result for the suppression of the superconducting transition temperature we distinguish 
the high temperature, $T_{c}^{0}>g_{T} \delta$, and low temperature, $T_{c}^{0}<g_{T} \delta$, regimes: For $3 \mathrm{D}$ systems the suppression of the transition temperature is given by

$$
\frac{\Delta T_{c}}{T_{c}^{0}}=-\left\{\begin{array}{ll}
\frac{A}{\pi g_{T}} \ln ^{2} \frac{g_{T} E_{C}}{T_{c}^{0}}, & T_{c}^{0}>g_{T} \delta \\
\frac{A}{\pi g_{T}}\left[\ln ^{2} \frac{g_{T} E_{C}}{T_{c}^{0}}-\frac{1}{2} \ln ^{2} \frac{g_{T} \delta}{T_{c}^{0}}\right], & T_{c}^{0}<g_{T} \delta
\end{array},\right.
$$

while for granular films we obtain

$$
\frac{\Delta T_{c}}{T_{c}^{0}}=- \begin{cases}\frac{1}{6 \pi^{2} g_{T}} \ln ^{3} \frac{g_{T} E_{C}}{T_{c}^{0}}, & T_{c}^{0}>g_{T} \delta \\ \frac{1}{6 \pi^{2} g_{T}}\left[\ln ^{3} \frac{g_{T} E_{C}}{T_{c}^{0}}-\frac{1}{4} \ln ^{3} \frac{g_{T} \delta}{T_{c}^{0}}\right], & T_{c}^{0}<g_{T} \delta\end{cases}
$$

Here $A=g_{T} a^{3} \int d^{3} q /(2 \pi)^{3} \varepsilon_{\mathbf{q}}^{-1}$ is the dimensionless constant where $\varepsilon_{\mathbf{q}}=2 g_{T} \sum_{\mathbf{a}}(1-\cos \mathbf{q a})$ with $\{\mathbf{a}\}$ being the lattice vectors. Equations (3) hold at temperatures $T_{c}^{0}-T_{c} \ll T_{c}^{0}$.

Turning to the discussion of the results (3), we note that the existence of two qualitatively different temperature regimes $\left(T>g_{T} \delta\right.$ and $\left.T<g_{T} \delta\right)$ is not surprising in view of the results for corrections to conductivity obtained in Ref. 8], where it was shown that the temperature dependence of the conductivity at $T<g_{T} \delta$ is dominated by the contribution from coherent electron motion at large distances, while at $T>g_{T} \delta$ the conductivity behavior is controlled by the scales of the order of the grain size. From Eq. (3a) one can see that the logarithmic corrections appear even in $3 D$ case; this property can serve as a specific characteristic of granular metals. In the high temperature regime $T>g_{T} \delta$ similar logarithmic corrections (with the same argument but different powers) were found in the corrections to the conductivity and density of states [7, 8]. Yet, the result for the critical temperature suppression in the high temperature regime depends on the dimensionality and is not universal contrary to the logarithmic corrections to conductivity found in the same regime in Ref. 7, 8]. We see that in granular superconducting films the suppression of the transition temperature is much stronger than that in $3 D$ granular samples. The extra logarithm power in two dimensions is due to the contribution of low momenta $q \ll a^{-1}$.

Our classification for $3 D$ granular superconductors can be derived from the following arguments: The critical conductance $g_{T}^{*}$ is defined by the condition that the correction (3a) becomes of the order of unity at $g_{T} \approx g_{T}^{*}$. Considering first the case where $g_{T}^{*}<g_{T}^{C}$, we see that the system should be a superconductor at $g_{T}>g_{T}^{*}$, since the conductance renormalization due to the effect of Coulomb interaction $\Delta g_{T}$ taken at the temperature $T \sim T_{c}^{0}$ is smaller than $g_{T}$ for all $g_{T}$ larger than $g_{T}^{*}$. Indeed $\Delta g_{T}=(1 / 6 \pi) \ln \left(g_{T} E_{C} / T_{c}^{0}\right)<g_{T}^{*}$. On the other hand, if $g_{T}<g_{T}^{*}$, then the interaction in the Cooper channel is completely suppressed and the system becomes an insulator at zero temperature because $g_{T}<g_{T}^{C}$. Thus in the case $g_{T}^{*}<g_{T}^{C}$ depending on the value of conductance $g_{T}$ one observes either insulating, at $g_{T}<g_{T}^{*}$, or

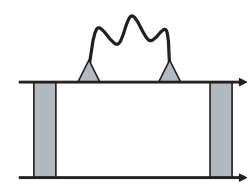

a)
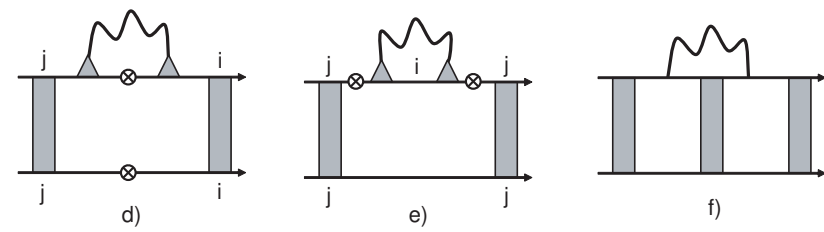

FIG. 1: Diagrams describing the first order self-energy corrections, $\delta \lambda_{1}$ to the Cooperon channel coupling constant $\lambda$ of granular metals due to the electron-electron repulsion. The solid lines denote the propagator of electrons, the wave lines describe screened Coulomb interaction and the dashed lines describe the elastic interaction of electrons with impurities. The black rectangle and triangle denote the renormalized Cooperon, see Eq. (5), and impurity vertex of granular metals respectively. The indices $i$ and $j$ stands for the number of the grains. The tunneling vertices are described by the circles. All diagrams should be taken with a factor of 2 . The final result for the sum of the diagrams (a) - (f) is given by Eq. (7).

superconducting, at $g_{T}>g_{T}^{*}$, phases at zero temperature. The second case, where $g_{T}^{*}>g_{T}^{C}$, is qualitatively different due to appearance of the intermediate metallic phase: In the region $g_{T}>g_{T}^{*}$ the system is a superconductor, in the region $g_{T}^{*}>g_{T}>g_{T}^{C}$ the interaction in the Cooper channel is completely suppressed, and the system is metallic since $g_{T}>g_{T}^{C}$, and finally in the region $g_{T}<g_{T}^{C}$ the system becomes an insulator.

Now we turn to the quantitative description of our model and derivation of Eq. (3a): We consider a $d$-dimensional array of superconducting grains in the metallic state. The motion of electrons inside the grains is diffusive and they can tunnel between grains. We assume that in the absence of the Coulomb interaction, the sample would have been a good metal at $T>T_{c}$.

The system of weakly coupled superconducting grains is described by the Hamiltonian

$$
\hat{H}=\hat{H}_{0}+\hat{H}_{c}+\hat{H}_{t} .
$$

The term $\hat{H}_{0}$ in Eq. (4a) describes isolated disordered grains with an electron-phonon interaction

$\hat{H}_{0}=\sum_{i, k} \epsilon_{i, k} a_{i, k}^{\dagger} a_{i, k}-\lambda \sum_{i, k, k^{\prime}} a_{i, k}^{\dagger} a_{i,-k}^{\dagger} a_{i,-k^{\prime}} a_{i, k^{\prime}}+\hat{H}_{i m p}$,

where $i$ stands for the number of the grains, $k \equiv(\mathbf{k}, \uparrow)$, $-k \equiv(-\mathbf{k}, \downarrow) ; \lambda>0$ is the interaction constant; $a_{i, k}^{\dagger}\left(a_{i, k}\right)$ are the creation (annihilation) operators for an electron in the state $k$ of the $i$-th grain and $\hat{H}_{i m p}$ describes elastic 
interaction of electrons with impurities. The term $\hat{H}_{c}$ in Eq. (4a) describes the Coulomb repulsion inside and between the grains and is given by

$$
\hat{H}_{c}=\frac{e^{2}}{2} \sum_{i j} \hat{n}_{i} C_{i j}^{-1} \hat{n}_{j},
$$

where $C_{i j}$ is the capacitance matrix and $\hat{n}_{i}$ is the operator of electrons number in the $i$-th grain. The last term in the right hand side of Eq. (4a) is the tunneling Hamiltonian

$$
\hat{H}_{t}=\sum_{i j, p, q} t_{i j} a_{i, p}^{\dagger} a_{j, q}
$$

where $t_{i j}$ is the tunneling matrix element corresponding to the points of contact of $i$-th and $j$-th grains.

The transition temperature, $T_{c}$ of the granular metals is determined from the pole of the superconducting propagator 14]. As in the case with homogeneous superconducting metals in the absence of the electron-electron repulsion the transition temperature of granular metals is independent of disorder.

To study the suppression of the transition temperature in granular metals we take into account the electronelectron repulsion in the Cooper channel. The first order correction $\delta \lambda$ to the Cooperon channel coupling constant $\lambda$ due to electron-electron repulsion is given by the diagrams in Figs. 1 and 2. These diagrams contain essentially the averaged one-particle Green functions (solid lines), effective screened Coulomb propagators (wave lines) and dashed lines that describe the elastic interaction of electrons with impurities. In the regime under consideration all characteristic energies are less than Thouless energy $E_{T}=D / a^{2}$, where $D$ is the diffusion coefficient of a single grain. This allows us to use the zero dimensional approximation for a single grain diffusion and Cooperon propagators. The electron hopping between the grains can be included using the diagrammatic technique developed in Refs. 5, 6. The complete expression for the renormalized Cooperon of granular metals (black rectangle in Fig. 1 and 2) has the following form

$$
C\left(\omega_{n}, \mathbf{q}\right)=\left(2 \pi \nu \tau^{2}\right)^{-1}\left(\left|\omega_{n}\right|+\varepsilon_{\mathbf{q}} \delta\right)^{-1},
$$

where $\mathbf{q}$ is the quasi-momentum, $\omega_{n}=2 \pi T n$ is the bosonic Matsubara frequency and $\nu$ is the density of states on the Fermi surface. The parameter $\varepsilon_{\mathbf{q}}$ in the right hand side of Eq. (5) appears due to the electron tunneling from grain to grain, it was defined below Eq. (31).

Deriving the analytical result for the diagrams in Figs. 1 and 2 it is important to take into account the fact that the single electron propagator itself gets renormalized due to electron hopping. Tunneling processes give rise an additional term to the self-energy part of the single electron propagator, see Fig. 3

$$
\tau^{-1}=\tau_{0}^{-1}+2 d g_{T} \delta,
$$

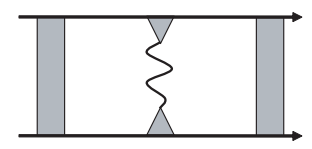

a)

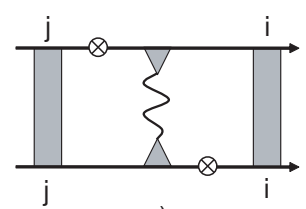

c)

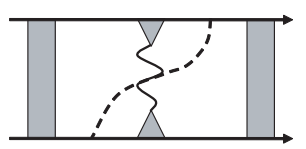

b)

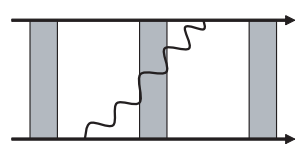

d)
FIG. 2: Diagrams describing the first order vertex corrections, $\delta \lambda_{2}$ to the Cooperon channel coupling constant $\lambda$ of granular metals due to the electron-electron repulsion. All notations are the same as in Fig. 1. The diagrams (b) - (d) should be taken with additional factor of 2 . After that the final result for the sum of the diagrams (a) - (d) is given by Eq. (10).

where $\tau_{0}$ is the unrenormalized electron mean free time and $g_{T}=2 \pi t^{2} \nu^{2}$ is the dimensionless tunneling conductance. Although the second term in the right hand side of Eq. (6) is much smaller than the first one it is important to keep it because the leading order contribution in $\tau_{0}^{-1}$ to the coupling constant $\lambda$ cancels ( see Eqs. (7) and (10) below).

We consider the contributions from diagrams in Fig. 1 and 2 separately. Using the result of Eq. (6) it is straightforward to check that the sum of the diagrams (a)-(f) in Fig. 1 results in the following correction, $\delta \lambda_{1}$ to the Cooperon channel coupling constant

$$
\begin{aligned}
& \frac{\delta \lambda_{1}}{\lambda}=-\pi T^{2} \sum_{\mathbf{q}}\left[\sum _ { \varepsilon _ { n } ( \varepsilon _ { n } - \Omega _ { n } ) < 0 } \left(\frac{2 V\left(\Omega_{n}, \mathbf{q}\right)}{\left|\varepsilon_{n}\right|\left(\left|\Omega_{n}\right|+\varepsilon_{\mathbf{q}} \delta\right)^{2}}\right.\right. \\
& \left.\left.+\frac{V(\Omega, \mathbf{q})}{\varepsilon_{n}^{2}\left(\left|\Omega_{n}\right|+\varepsilon_{\mathbf{q}} \delta\right)}\right)-\sum_{\varepsilon_{n}\left(\varepsilon_{n}-\Omega_{n}\right)>0} \frac{V\left(\Omega_{n}, \mathbf{q}\right)}{\varepsilon_{n}^{2}\left(\left|2 \varepsilon_{n}-\Omega_{n}\right|+\varepsilon_{\mathbf{q}} \delta\right)}\right] .
\end{aligned}
$$

Here the summation is going over the quasi-momentum, $\mathbf{q}$ and over the fermionic, $\varepsilon_{n}=\pi T(2 n+1)$ and bosonic, $\Omega_{n}=2 \pi T n$ Matsubara frequencies. The propagator of the screened electron-electron interaction, $V\left(\Omega_{n}, \mathbf{q}\right)$ in Eq. (7) is given by the expression

$$
V\left(\Omega_{n}, \mathbf{q}\right)=\frac{2 E_{C}(\mathbf{q})\left(\left|\Omega_{n}\right|+\varepsilon_{\mathbf{q}} \delta\right)}{4 \varepsilon_{\mathbf{q}} E_{C}(\mathbf{q})+\left|\Omega_{n}\right|} .
$$

The parameter $\varepsilon_{\mathbf{q}}$ in Eqs. (7) and (8) was defined below Eq. (3a). The charging energy $E_{C}(\mathbf{q})=e^{2} / 2 C(\mathbf{q})$ in Eq. (8) is expressed in terms of the Fourier transform of the capacitance matrix $C(\mathbf{q})$ which has the following asymptotic form

$$
C^{-1}(\mathbf{q})=\frac{2}{a^{D}} \begin{cases}\pi / q & D=2 \\ 2 \pi / q^{2} & D=3 .\end{cases}
$$




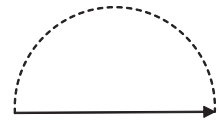

a)

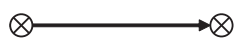

b)
FIG. 3: Diagrams describing self-energy corrections to the single electron propagator due to a) elastic interaction of electrons with impurities and b) electron hopping. The solid lines denote the bare propagator of electrons and the dashed line describes the elastic interaction of electrons with impurities. The tunneling vertices are described by the circles.

To obtain the total correction, $\delta \lambda$ to the Cooperon channel coupling constant $\lambda$ due to electron-electron interaction the diagrams in Fig. 2 that represent vertex corrections should be taken into account. These diagrams results in the following contribution

$$
\begin{aligned}
\frac{\delta \lambda_{2}}{\lambda}= & -\pi T^{2} \sum_{\mathbf{q}}\left[\sum_{\varepsilon_{n}\left(\varepsilon_{n}-\Omega_{n}\right)<0} \frac{V\left(\Omega_{n}, \mathbf{q}\right)}{\left|\varepsilon_{n}\right|\left|\varepsilon_{n}-\Omega_{n}\right|\left(\left|\Omega_{n}\right|+\varepsilon_{\mathbf{q}} \delta\right)}\right. \\
& \left.+\sum_{\varepsilon_{n}\left(\varepsilon_{n}-\Omega_{n}\right)>0} \frac{V\left(\Omega_{n}, \mathbf{q}\right)}{\left|\varepsilon_{n}\right|\left|\varepsilon_{n}-\Omega_{n}\right|\left(\left|2 \varepsilon_{n}-\Omega_{n}\right|+\varepsilon_{\mathbf{q}} \delta\right)}\right]
\end{aligned}
$$

Using Eqs. (8), (9) and summing over the frequencies $\varepsilon_{n}$, $\Omega_{n}$ and quasi-momentum, $\mathbf{q}$ in Eqs. (7) and (10) with the logarithmic accuracy we obtain the final result (3) for the superconducting transition temperature in granular metals.

In conclusion, we considered the suppression of superconducting transition temperature due to Coulomb repulsion in granular metals. We found the correction to the transition temperature for $3 D$ granular samples and films at large tunneling conductance between the grains. We demonstrated that the suppression of superconductivity in $3 D$ samples can be characterized by the critical value of the conductance $g_{T}^{*}$ introduced in Eq. (2) such that for samples with $g_{T}<g_{T}^{*}$ the superconductivity is suppressed. Taking into account effects of Coulomb blockade of conductivity that become essential for samples with $g_{T}<g_{T}^{C}$, where the critical value $g_{T}^{C}$ is given by Eq. (11) we introduced the classification of $3 D$ granular samples composed from superconducting grains according to the possibility of having insulating, metallic or superconducting phases at zero temperature.

We would like to thank A. I. Larkin for useful discussions. This work was supported by the U.S. Department of Energy, Office of Science through contract No. W-31109-ENG-38.

[1] K. L. Ekinci and J. M. Valles, Jr., Phys. Rev. Lett. 82, 1518 (1999).

[2] A. Gerber et al, Phys. Rev. Lett. 78, 4277 (1997).

[3] H. M. Jaeger, D. B. Haviland, A. M. Goldman, and B. G. Orr, Phys. Rev. B 34, 4920 (1986).

[4] R. W. Simon et al, Phys. Rev. B 36, 1962 (1987).

[5] I. S. Beloborodov and K. B. Efetov, Phys. Rev. Lett. 82 , 3332 (1999).

[6] I. S. Beloborodov, K. B. Efetov, A. Altland and F. W. J. Hekking, Phys. Rev. B 63 , 115109 (2001).

[7] K. B. Efetov and A. Tschersich, Europhys. Lett. 59, 114, (2002); Phys. Rev. B 67, 174205 (2003).

[8] I. S. Beloborodov, K. B. Efetov, A. V. Lopatin and V. M. Vinokur, cond-mat/0304448

[9] Yu. N. Ovchinnikov, Sov. Phys. JETP 37, 366 (1973).

[10] S. Maekawa and H. Fukuyama, J. Phys. Soc. Jpn. 51, 1380 (1982); S. Maekawa, H. Ebisawa and H. Fukuyama, J. Phys. Soc. Jpn. 52, 1352 (1983).

[11] A. M. Finkelstein, Sov. Phys. JETP Lett. 45, 46 (1987); Phisica B 197, 636 (1994).

[12] A. I. Larkin, Ann. Phys. 8, 785 (1999).

[13] B. L. Altshuler and A. G. Aronov, in Electron-Electron Interaction in Disordered Systems, ed. by A. L. Efros and M. Pollak, North-Holland, Amsterdam (1985).

[14] A. A. Abrikosov, L. P. Gorkov and I. E. Dzyaloshinskii, Methods of Quantum Field Theory in Statistical Physics (Prentice-Hall, Englewood Cliffs, NJ, 1963). 